\title{
The influence of tea catechins on immune function in the elderly: application of the Bayesian statistical approach to an exploratory clinical trial
}

\section{Ryo Iketani ${ }^{1}$, Daisuke Furushima ${ }^{1}$, Takuma Nishimura', Yui Hirama ${ }^{2}$, Shintaro Onishi ${ }^{2}$ Takuya Mori ${ }^{2}$, Yuko Ohno ${ }^{3}$, Hiroshi Yamada ${ }^{1}$}

${ }^{I}$ Drug Evaluation and Informatics, Univecrsity of Shizuoka, Japan, ${ }^{2}$ Biological Science Laboratories, Kao Corporation, Japan, ${ }^{3}$ Mathematical Health Science, Osaka University, Japan

Background. The frequentist statistical approach, used to analyze the effects of intervention, often overlooks significant differences. This occurs because the statistical framework depends on the study sample size or whether p-values from hypothetical tests are below a defined significance level. The Bayesian statistical approach, which can directly calculate probabilities of interesting differences, may be an alternative useful choice for generating new hypotheses in exploratory studies. Therefore, we used data from a previous study and applied the Bayesian statistical approach to assess its utility in exploratory clinical trials.

Methods. Results from a single-arm, open-label clinical trial investigating the influence of tea catechins on immune function in 20 elderly subjects were re-analyzed using the Bayesian hierarchical model. The trial measured the following values as changes from the baseline: natural killer (NK) cell activity; granzyme B and perforin in serum; and secretion of interferon-alpha, -gamma, interferon-inducible protein (IP)-10, interleukin (IL)-10, and -12 from peripheral blood mononuclear cells stimulated by TLR-7/8 agonist (R-848). Using the frequentist framework and paired t-tests, NK cell activity and the amount of interferon-alpha were determined to be significantly increased after the intervention. Other measurements changes were not deemed statistically significant. We then calculated the Bayesian p-values (\%), in which change values are higher than 0 , to add further interpret the obtained data.

Results. The Bayesian p-values for NK cell activity and interferon-alpha were $100.0 \%$ and 95.9\%, respectively. Additionally, the p-value for IP-10 was $96.9 \%$, and for IL-12 was $99.7 \%$. The p-values for interferon-gamma and IL-10 were moderately high $(92.8 \%$ and $84.9 \%$, respectively). The p-value regarding perforin was slightly high (68.7\%), while the p-value for granzyme B was moderately low (17.9\%).

Conclusions. Applying the Bayesian statistical approach, we found that IP-10 and IL-12 may be increased by the intervention despite the limited sample size. Additionally, this approach shows that the intervention may also influence interferon-gamma and IL-10 expression. These results would provide useful information in further studies when endpoints are set. Therefore, a combination of the frequentist and Bayesian approaches would be useful when analyzing data from exploratory clinical trials. 